\title{
A Novel Design of Magnetorheological Damper with Annular Radial Channel
}

\author{
Shisha Zhu, Libo Tang, Jingang Liu, Xinzi Tang, and Xiongwei Liu \\ School of Mechanical Engineering, Xiangtan University, Xiangtan 411105, China \\ Correspondence should be addressed to Libo Tang; 834483655@qq.com
}

Received 14 May 2015; Revised 6 August 2015; Accepted 6 September 2015

Academic Editor: Rafał Burdzik

Copyright (C) 2016 Shisha Zhu et al. This is an open access article distributed under the Creative Commons Attribution License, which permits unrestricted use, distribution, and reproduction in any medium, provided the original work is properly cited.

With the development of automotive vibration technology, the semiactive suspension system with adjustable damping force and high reliability is taken seriously. The magnetorheological damper (MRD) that applies intelligent material (magnetorheological fluid) is the key element of this system. It can achieve a continuous and adjustable damping and then reaches the purpose of comfort. In order to improve the damping effect of MRD, this paper presents a MRD, which has magnetorheological (MR) effect along annular radial channel. The paper completely designs the structure and magnetic circuit of MRD. Based on the theory of electromagnetism and MR fluid dynamics, the paper analyzes and tests the external characteristics of the MRD by the MATLAB/Simulink and the vibration experiment. The results compared with ordinary MRD reveal that the damping force obviously increases and has wide adjustable range, thus verifying the reasonableness of the damper design.

\section{Introduction}

Smart structure system is an active research topic in the contemporary. The MRD takes MR fluid as working medium. It has excellent electromagnetic controllability, provides good electrical-mechanical coupling interface for smart structure system, and possesses broad developmental prospect in the fields of aircraft, automobile, construction, high-speed trains, and so forth. For meeting the requirements of damping force, the design of MRD demands generating enough magnetic field in the work region. The magnetic field intensity is restricted by the magnetic materials and magnetic circuit. Therefore, in order to increase MR effect, the reasonable structure design of magnetic circuit is an important research content in MRD.

In the past 20 years, the MRD with different magnetic circuit structures was studied by scholars and applied to the semiactive control system $[1,2]$. The semiactive control system based on MRD has the advantages of continuously adjustable damping, low power consumption, simple structure, high reliability, easy maintenance, and so forth. It is being the mainstream development direction of vibration control. In 1994, Carlson and Chrzan [3] developed a commercial MRD of annular flowing channel (RD-1005-3, LORD
Corporation), which marks the arrival of application about MR damping technology. However, the volume of the fluid in the MRD is up to 5 litres. To the problem that the cylinder volume is too large, Bai et al. [4] proposed a MRD using bypass valve; it has the characteristic that the damping is big and effectively reduces the cylinder volume but occupies installation space. In addition, Mao et al. [5] proposed a MRD using bifold valve, which is, respectively, installed on both ends of cylinder. Although the bifold valve improves the effective length of generating MR effect, it increases the complexity of the damper structure. Yazid et al. [6] proposed a MRD of mixing work mode based on shear and squeeze, which can generate larger damping force and magnetic field but only has a short stoke under mixing mode. In addition, Meng et al. [7] researched the typical circuit structure of MRD and, respectively, analyzed the influence of magnetic length, core section, and cylinder thickness on the magnetic field. Zheng et al. [8] and other scholars made a deeper study, who found that magnetic field generated by two reverse winding coils can considerably exceed magnetic field generated by single winding coil in damping channel, but the effective damping channel is shorter. Therefore, the design has to increase the volume of magnetic circuit to enhance magnetic field intensity in damping channel. It influences coil 
layout and limits the utilization rate of the magnetic field in damper.

At present, the damping channel is generally the axial channel of the piston edge in MRD. The structure makes heat produced by electromagnetic coil unable to be timely dissipated, thereby raising the temperature of MR fluid in the channel, thus exacerbating the influence from temperature on MR effect. Furthermore, the region generating MR effect is between the piston and cylinder, which easily causes noncollinearity of the piston rod and cylinder axis in the reciprocating motion, so that the gap width of occurring $M R$ effect is not equal, thereby resulting in getting stuck piston because of different axial damping force. Besides, in order to increase the effective length of generating the MR effect and improve the utilization rate of magnetic field, increasing axial length of the piston and number of the coils is commonly used. However, it observably shortens the working stroke of the damper on condition of uniform installation size. To solve these problems, this paper presents a MRD with annular radial channel. The damper can observably promote channel length to generate MR effect but will not change the width of gap for moving eccentric piston. Therefore, it effectively avoids the defects in prestudying MRD. At the same time, to obtain the damping force-stroke and the damping forcevelocity property of the MRD, this paper analyzed the external characteristic of the damper with simulation method and the reasonableness based on simulation and experiment results.

\section{The Principle and Design of MR Damper}

2.1. The Principle of Damper. Figure 1 illustrates the design concept of the MRD with the inner channel. The MR effect occurs between the middle magnetizer and the upper (under) magnetizer of piston. The MR fluid inflows the piston from the central hole of upper or under magnetizer, generates MR effect at the channel gap, and then outflows from the under or upper magnetizer.

The MRD using the inner channel is compared with the ordinary MRD using the external channel. Its working stroke is increased and space occupied by piston is decreased when the whole damping force is the same. At the same time, it can effectively increase the area of generating MR effect and the damping force on the same conditions.

2.2. The Structural Design of Damper. The structural design of the MRD mainly includes design of the cylinder, the piston rod, the electromagnetic piston, the guide seat, and the gas compensative chamber. The cylinder acts as a key component in the MRD. It not only bears extrusion produced by movement but also is the main device of dissipating electromagnetic heat and friction heat in work. The cylinder diameter can be determined by the maximum damping force and the allowable pressure of cylinder:

$$
D=\sqrt{\frac{4 F_{\max }}{\pi p\left(1-\lambda^{2}\right)}},
$$

where $p$ is allowable pressure of cylinder, $F_{\max }$ is maximum damping force of damper, and $\lambda$ is the ratio given by $d / D$, generally taken to be $0.3 \sim 0.35$ [9].

The piston rod is a major component bearing force in the MRD. It directly connects to the suspension and delivers the impact produced by vehicle body, so the piston rod not only needs to have good mechanical properties but also must have sufficient strength. Moreover, the wire hole is opened on the centre of piston rod to conveniently wind coil:

$$
\begin{aligned}
& d=\lambda D, \\
& d>\sqrt{\frac{4 F_{\max }}{\pi[\sigma]}+d_{0}^{2}} .
\end{aligned}
$$

Here, $[\sigma]$ is the yield stress of material. $d_{0}$ is the diameter of wire guide hole. In order not to change work gap for eccentric movement of piston, the design of the guide seat must ensure coaxial degree of the cylinder and the piston rod, so the guide seat must have enough length:

$$
H \geq \frac{S}{2}+\frac{D}{2},
$$

where $S$ is the working stroke of damper.

Electromagnetic piston is the heart of structure and magnetic circuit design in the MRD. Its structure can affect the magnetic field distribution and the properties of damper. Therefore, the design of the electromagnetic piston needs to fully enhance axial magnetic field under constant size of piston, which is shown in Figure 2.

The gas compensative chamber is to compensate volume difference generated by upper and under chambers during movement of the MRD piston. It should have enough compensation volume:

$$
V=\frac{\pi}{4} D^{2} h \geq \frac{\pi}{4} d^{2} S .
$$

Here, $h$ is the length of compensation chamber.

2.3. The Design of Magnetic Circuit. The magnetic circuit design is a critical issue for damping effect of MRD. Because of ordinary $\mathrm{MRD}$, the defects that are low utilization rate of magnetic field and magnetic saturation exist. This paper promotes utilization rate of magnetic field as goal to design magnetic circuit, whose structure is shown in Figure 3.

According to the magnetic circuit shown in Figure 3, which can be divided into three areas, utilizing the basic theory that calculates reluctance can, respectively, obtain magnetic resistance of area 1 , area 2 , and area 3 :

$$
\begin{aligned}
R_{m 1} & =\frac{l-2 b}{\mu_{0} \mu_{1}(\pi / 4) c^{2}}, \\
R_{m 2} & =\frac{D-r_{1}}{\mu_{0} \mu_{2} \pi\left(\left(D+r_{1}\right) / 2\right) b}, \\
R_{m 3} & =\frac{l-b}{\mu_{0} \mu_{2} \pi(D+a) a} .
\end{aligned}
$$




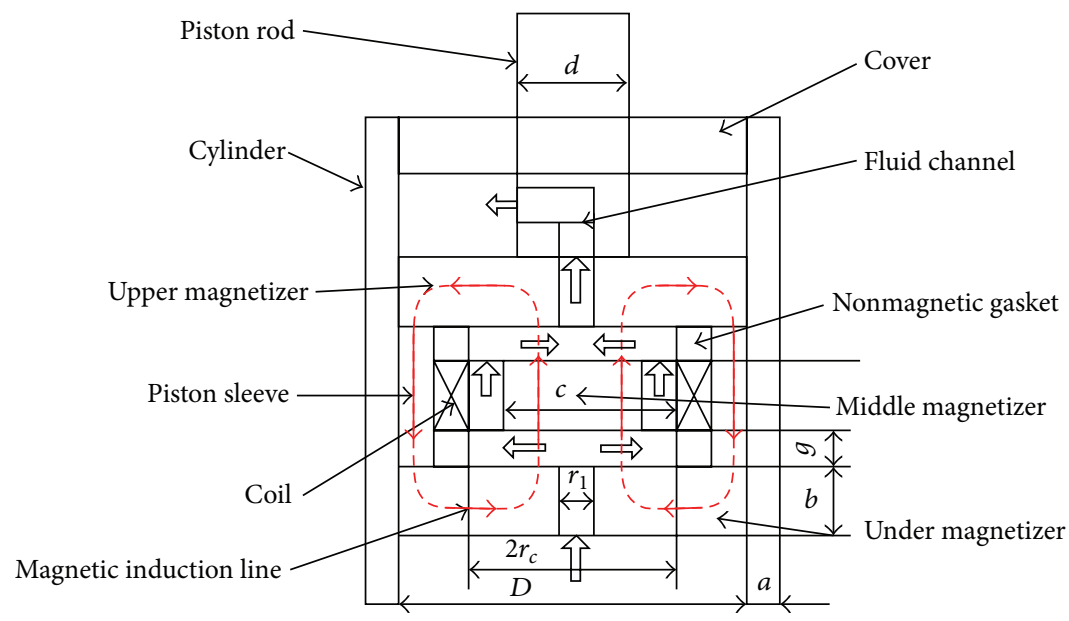

FIgURE 1: The structure principle of damper with the type of inner channel.

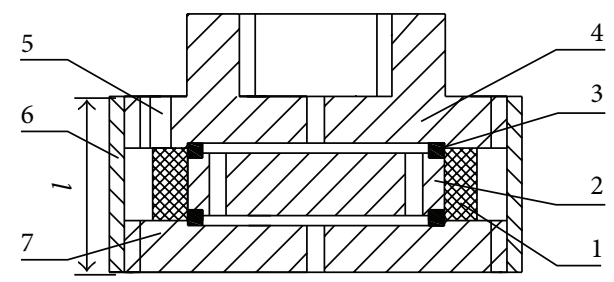

FIGURE 2: The electromagnetic piston with combined type: (1) coil, (2) middle magnetizer, (3) gasket, (4) upper magnetizer, (5) wire hole, (6) piston sleeve, and (7) under magnetizer.

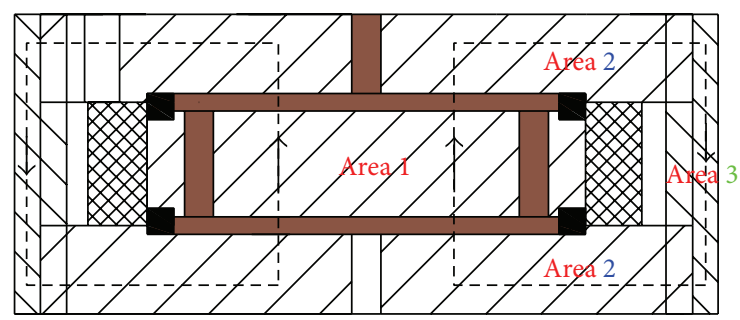

FIgURE 3: The structure of magnetic circuit.

Similarly, the magnetic resistance of channel gap is given by

$$
R_{\mathrm{MR}}=\frac{g}{\mu_{0} \mu_{\mathrm{MR}}(\pi / 4) c^{2}},
$$

where $\mu_{1}, \mu_{2}$, and $\mu_{\mathrm{MN}}$, respectively, are relative permeability of electrical iron, piston magnetizer, and MR fluid. $\mu_{0}$ is the vacuum permeability. $a$ is the thickness of piston sleeve. $b$ is the height of upper magnetizer (under magnetizer). $c$ is the diameter of middle magnetizer. $l$ is the length of piston. The total magnetomotive force is

$$
F=N I=\phi\left(R_{m 1}+R_{m 2}+R_{m 3}+R_{\mathrm{MR}}\right) .
$$

The represented above-obtained reluctance in (7) can calculate turns of coil as follows:

$$
N=\frac{\phi\left(R_{m 1}+R_{m 2}+R_{m 3}+R_{\mathrm{MR}}\right)}{I} .
$$

Figure 4 depicts assembly diagram of the MRD with annular radial channel. The basic structure includes cylinder, electromagnetic piston and floating piston of combinative type, bottom cover, and piston rod. In the damper, the under ring fixes with bottom cover, and the upper ring connects with piston rod. The MRD is installed on the vehicle suspension by the upper and under rings. When vehicle produces the vibration, the piston rod drives electromagnetic piston to compression or recovery. Because the shear yield stress of MR fluid can be adjusted by applied current, the damping force generated by damper can preferably improve riding performance of vehicle on different roads.

\section{The External Characteristic Analysis of MRD}

3.1. The Calculating Model of Damping Force. According to the structure of damper shown in Figure 4, the calculating model of damping force of MRD with annular radial channel is deduced based on Bingham constitutive model [10] as follows:

$$
\begin{aligned}
F & =\left[p_{a}\left(\frac{V_{0}}{V_{0}+A_{P} S}\right)+3 \frac{\tau_{y}}{h}\left(r_{2}-r_{1}\right) \operatorname{sign}(v)\right. \\
& \left.+\frac{6 Q \mu}{\pi h^{3}} \ln \left(\frac{r_{2}}{r_{1}}\right)+\frac{128 Q \mu}{\pi D^{4}} h_{a}\right]\left(A-A_{P}\right) \\
& -p_{a}\left(\frac{V_{0}}{V_{0}+A_{P} S}\right) A+F_{f} .
\end{aligned}
$$

Here, the first term is compensative chamber pressure in square brackets of (9), the second term is controllable pressure drop, the third term is viscous pressure drop, and the fourth term is flow pressure drop. $Q$ refers to the flow rate of volume. $r_{1}, r_{2}$ are the inner and outer diameters of magnetizer. $\tau_{y}$ is the shear yield strength of MR fluid. $S$ is the displacement of piston. $A$ and $A_{P}$ refer to the cross-sectional area of cylinder and the piston rod. $h$ is the gap width of channel. $p_{a}, V_{0}$, respectively, are the initial pressure and initial 


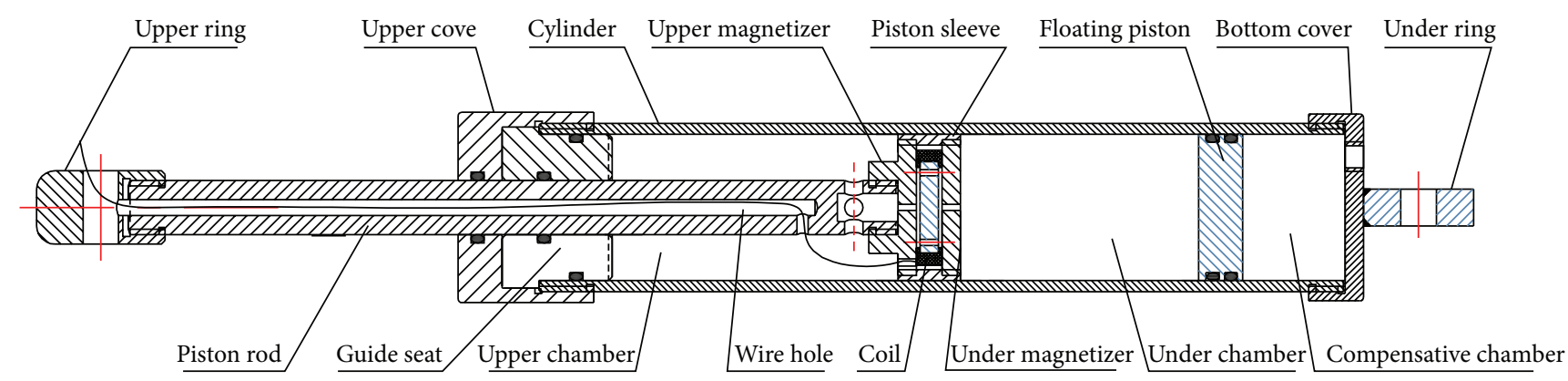

FIgURE 4: The assembly diagram of MR damper.

volume of compensative chamber. $F_{f}$ is the damping force generated by friction.

\subsection{The External Characteristic Simulation of Damper. The} performance of assessing damper includes damping forcestroke $(F-S)$ and damping force-velocity $(F-V)$ property. The slope of $F-V$ curve reflects the damping coefficient of damper, and the area surrounded by $F-S$ curve reflects the size of dissipating energy. Therefore, we usually take the two properties as important index to evaluate the performance of damper. According to the calculating model of damping force obtained by previous section, this paper establishes simulation model of damping force about the MRD based on MATLAB/Simulink shown in Figure 5.

The simulation model describes the relationship about damping force-velocity and damping force-displacement under different current $I$. We set that the displacement is sinusoidal excitation, the amplitude $A=75 \mathrm{~mm}$, and the frequency $f=1 \mathrm{~Hz}$. The applied current $(I)$, respectively, is taken as $0,0.5,1,1.5$, and $2 \mathrm{~A}$. The force-stroke $(F-S)$ and force-velocity $(F-V)$ curves are shown in Figure 6.

3.3. Results and Discussions. The damping effect of vehicle suspension is usually described by external characteristic of damper, such as the $F-S$ and $F-V$ characteristic curves. The area surrounded by the $F-S$ curve reflects the damping effect of damper in a vibration period. Figure 6(a) shows that the area encircled by $F-S$ curve gradually increases with increasing of electric current, which indicates that the damping force of MRD is controllable. And it shows that the damping force is asymmetric in the compression and extension stroke. The asymmetry is mainly produced by gas damping from compensative chamber. Besides, what we can see from the $F-S$ diagram is that the damping force of MRD may be controlled by adjusting applied current in stretching and compression stroke. Thus it can real-time optimally adjust the damping of the vehicle suspension according to road condition and improves good riding and stability performance. At the same time, Figure 6(a) presents that the damping force of MRD is about $1500 \mathrm{~N}$ as the applied current takes $0 \mathrm{~A}$. It is the uncontrollable damping force that mainly contains viscous force. When the applied current takes $2 \mathrm{~A}$, the damping force is about $3500 \mathrm{~N}$, in which the controllable damping force is about $2000 \mathrm{~N}$, namely, Coulomb force. Therefore, the adjustable range of damping force is [1500, 3500], and the adjustable coefficient can reach 1.33. This indicates that the MRD has large damping force and damping scope. Figure 6(b) shows that the damping force increases with increasing of velocity. When the applied current takes $0 \mathrm{~A}$, the $F-V$ curve approximately is straight line, which indicates that MR fluid presents Newton characteristic in the absence of magnetic field. When the applied current takes nonzero value, the $F-V$ curve is nonlinear, which indicates that MR fluid exhibits non-Newtonian characteristic under the effect of magnetic field.

\section{The Experimental Research of MRD}

4.1. The Vibration Test System of MRD. The vibration test bench of MRD is the device that can simulate vibration environment and test the mechanical properties. According to the basic structure and principle, it is generally divided into mechanical bench, electrohydraulic bench, and electric bench. Because of the electric bench's simple structure, easy manufacture, and low cost, this paper applies the self-designed electric bench to test external characteristic of MRD. Its basic structure includes drive device, sensor, and test device. The drive device consists of electromotor and slider crank mechanism, which generates sinusoidal vibration signal. The test system is shown in Figure 7.

4.2. The Experimental Results. Respectively inputting applied current $(I)$ as $0,0.5,1,1.5$, and $2 \mathrm{~A}$ and adjusting frequency inverter of the electromotor make drive device generate vibration signal. Through real-time data measurement, drawing of force-stroke and force-velocity curves is shown in Figure 8.

As shown in Figure 8(a), it is given that the damping force and the area encircled by $F-S$ curve increase with gradually enhancing current $(I)$. The maximum damping force is up to $4000 \mathrm{~N}$, which indicates that the damping effect of the MRD is good. Figure $8(b)$ is the force-velocity curve; when the velocity and applied current increase, the damping force gradually rises. This is because the speed changes viscous damping of MRF, and the current changes the shear yield stress of MRF. When the conditions are the same, the test results are consistent with the simulation curve, but there are also some differences. This is mainly because the slider crank 


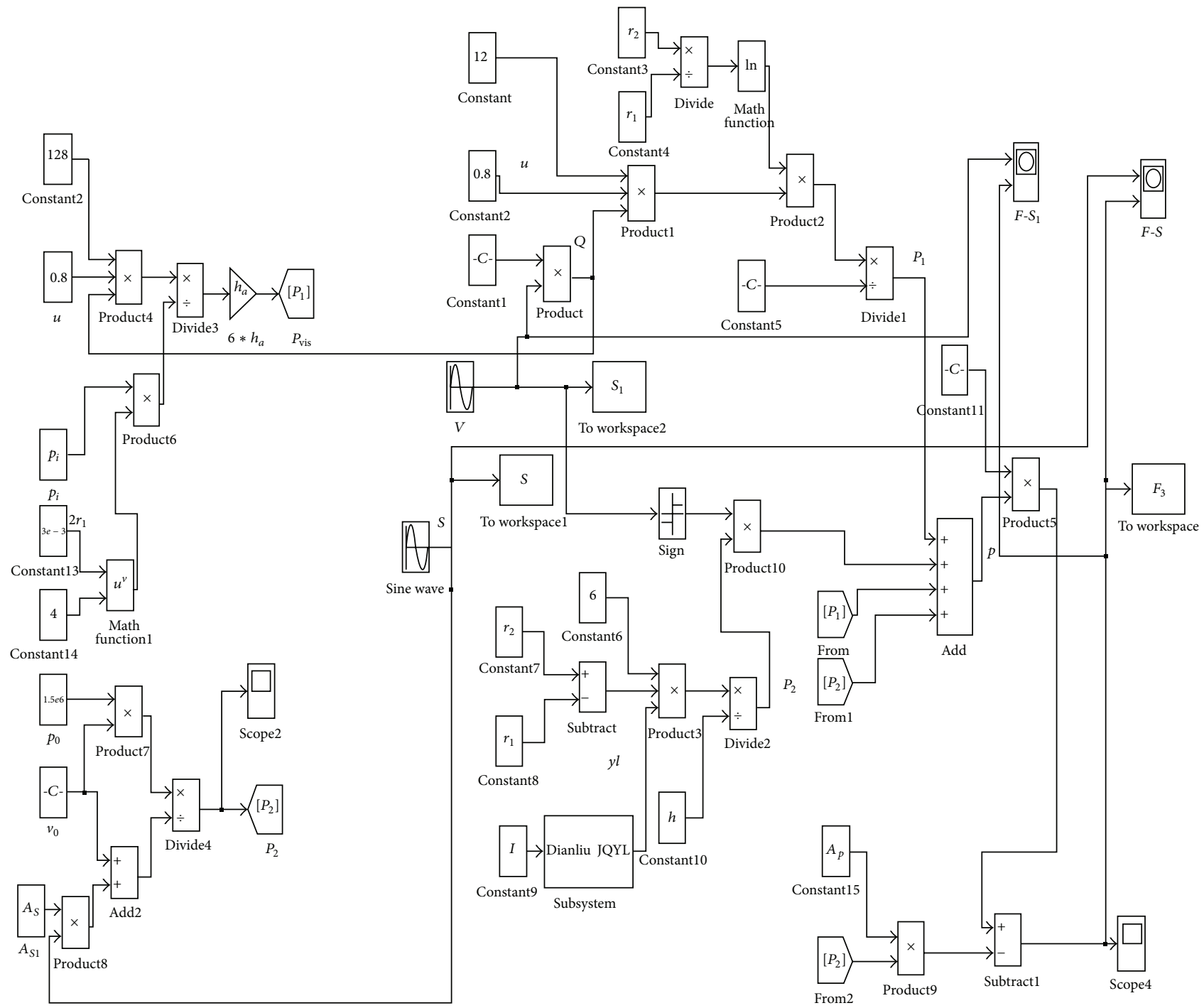

FIGURE 5: The external characteristic simulation mode of MR damper with the type of inner channel.

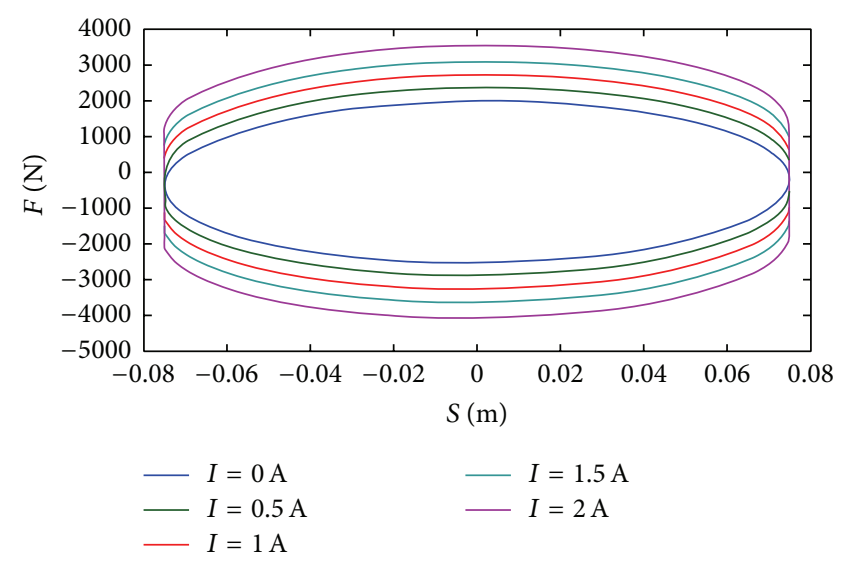

(a) The force-stroke curve

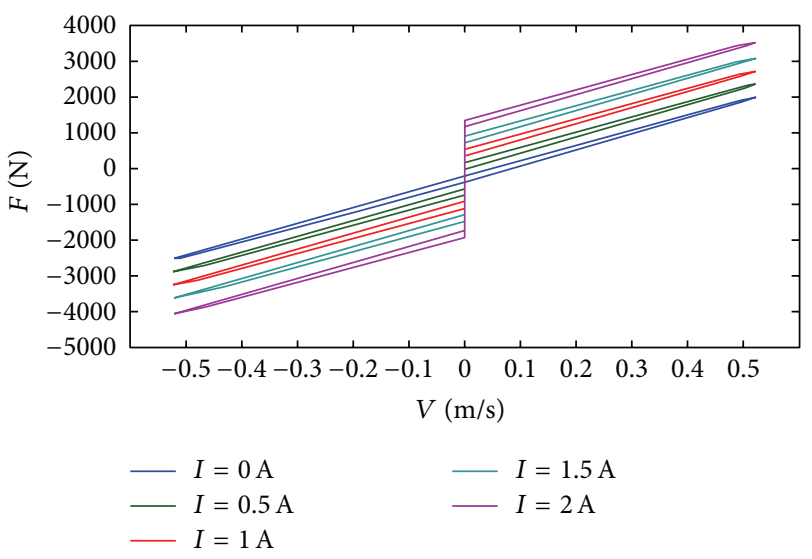

(b) The force-velocity curve

Figure 6: The $F-S$ and $F-V$ curves of MRD with annular radial channel. 


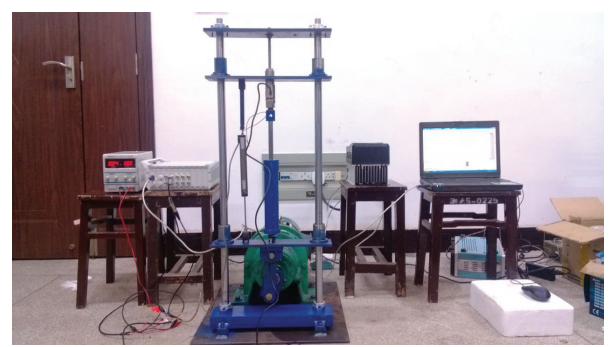

Figure 7: The vibration test system of MRD.

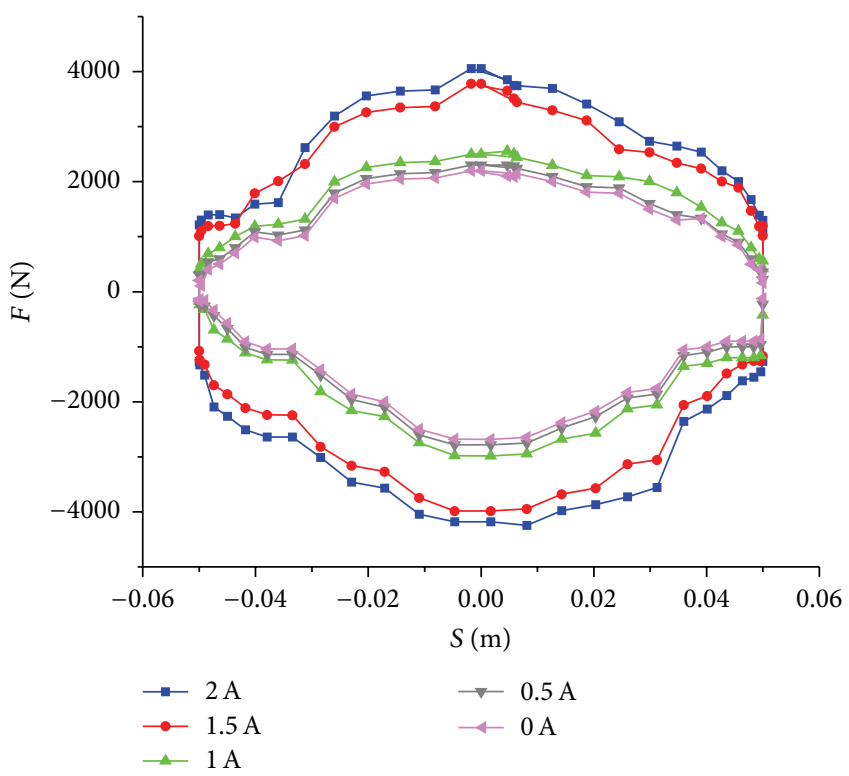

(a) The force-stroke test curve

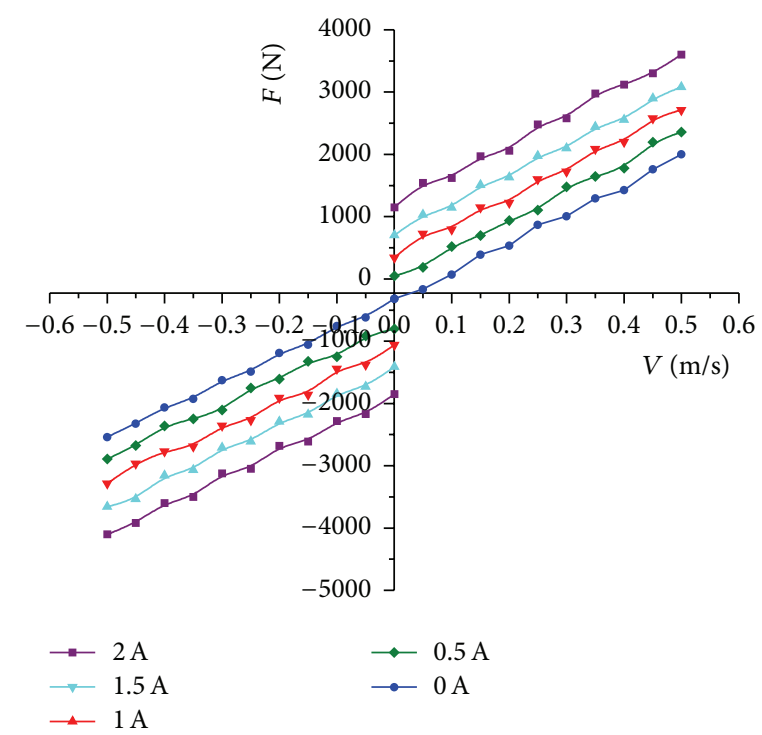

(b) The force-velocity test curve

FIGURE 8: The test curve of MRD with annular radial channel.

mechanism causes some fluctuations in movement, and the vibration test bench causes shape and position deviation in processing and assembling.

\section{Conclusions}

The MRD with annular radial channel is designed in this paper, whose fluid channel takes the type of inner channel, in which MR fluid inflows and outflows from the middle of piston. The design can effectively avoid the fact that the piston gets stuck in reciprocating motion because the axial force is uneven. Meanwhile, if we want to improve controllable damping force of the damper, it only requires to increase its radial size but does not affect axial working stroke. In addition, based on MATLAB/Simulink and the vibration test bench, we establish the external characteristic simulation model of the MRD, test the external characteristic by vibration experiment, analyze the performance index such as the damping force range and the adjustable coefficient, and so forth. With the increasing of applied current $I$, the damping force of MRD grows to $3500 \mathrm{~N}$ from $1500 \mathrm{~N}$, the $F$-S curve is smooth and plump, and the experimental results are consistent with the simulation curve. Therefore, this indicates that the MRD has large damping scope and good damping effect, can meet the damping force requirements of vehicle suspension, and can be applied to semiactive suspension system of the adjustable damping type.

\section{Conflict of Interests}

The authors declare that there is no conflict of interests regarding the publication of this paper.

\section{Acknowledgments}

The authors gratefully acknowledge the support provided by the National Natural Science Foundation of China (51075345 and 51475402) and the Natural Science Foundation of Hunan Province (BJJ8005). 


\section{References}

[1] K. H. Lam, Z. H. Chen, Y. Q. Ni, and H. L. W. Chan, "A magnetorheological damper capable of force and displacement sensing," Sensors and Actuators A: Physical, vol. 158, no. 1, pp. $51-59,2010$.

[2] M. N. Sheikh, J. Xiong, and W. H. Li, "Reduction of seismic pounding effects of base-isolated RC highway bridges using MR damper," Structural Engineering and Mechanics, vol. 41, no. 6, pp. 791-803, 2012.

[3] J. D. Carlson and M. J. Chrzan, "Magnetorheological fluid dampers," US Patent. No.5,277,281, 1994.

[4] X. X. Bai, W. Hu, and N. M. Wereley, "Magnetorheological damper utilizing an inner bypass for ground vehicle suspensions," IEEE Transactions on Magnetics, vol. 49, no. 7, pp. 34223425, 2013.

[5] M. Mao, W. Hu, Y.-T. Choi, and N. M. Wereley, "A magnetorheological damper with bifold valves for shock and vibration mitigation," Journal of Intelligent Material Systems and Structures, vol. 18, no. 12, pp. 1227-1232, 2007.

[6] I. I. M. Yazid, S. A. Mazlan, T. Kikuchi, H. Zamzuri, and F. Imaduddin, "Design of magnetorheological damper with a combination of shear and squeeze modes," Materials \& Design, vol. 54, pp. 87-95, 2014.

[7] Y. P. Meng, C.-R. Liao, H.-H. Zhang, and L.-Z. Mao, "Magnetic circhuit design and related problem studies of automotive magnetorheological damper," Journal of Functional Materials, vol. 5, no. 37, pp. 768-770, 2006.

[8] L. Zheng, L. Y. Nong, H. Yong et al., "Magnetic circuit design and structure optimization of magnetorheological damper," Journal of Vibration Engineering, vol. 21, no. 2, pp. 174-177, 2002.

[9] C. C. Zhou, The Design and Characteristic Simulation of Shock Absorber, China Machine Press, Beijing, China, 2012.

[10] P. J. Rankin, J. M. Ginder, and D. J. Klingenberg, "Electroand magneto-rheology," Current Opinion in Colloid \& Interface Science, vol. 3, no. 4, pp. 373-381, 1998. 


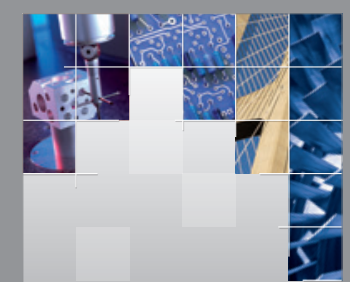

\section{Enfincering}
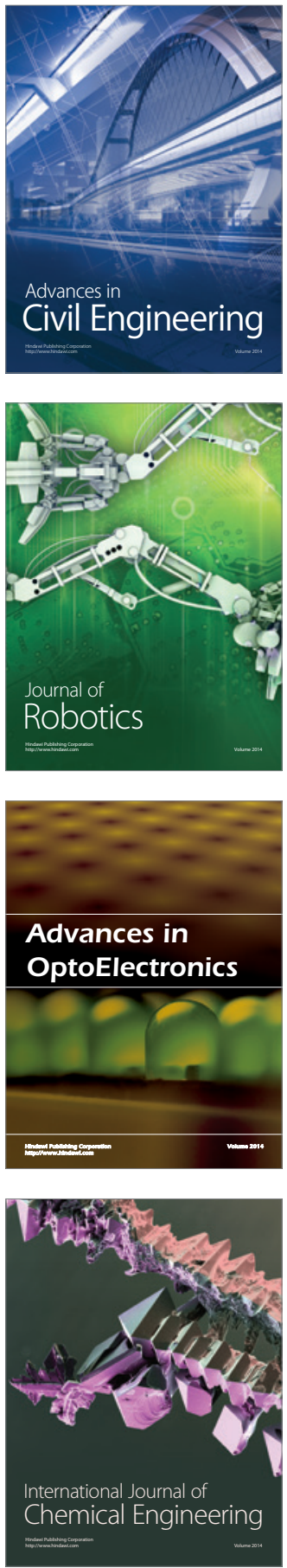

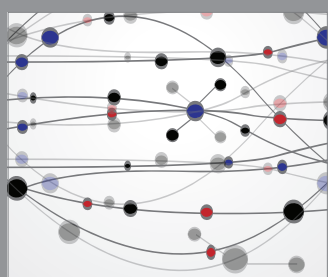

The Scientific World Journal

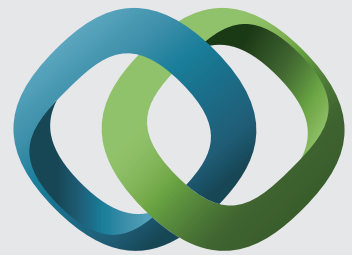

\section{Hindawi}

Submit your manuscripts at

http://www.hindawi.com
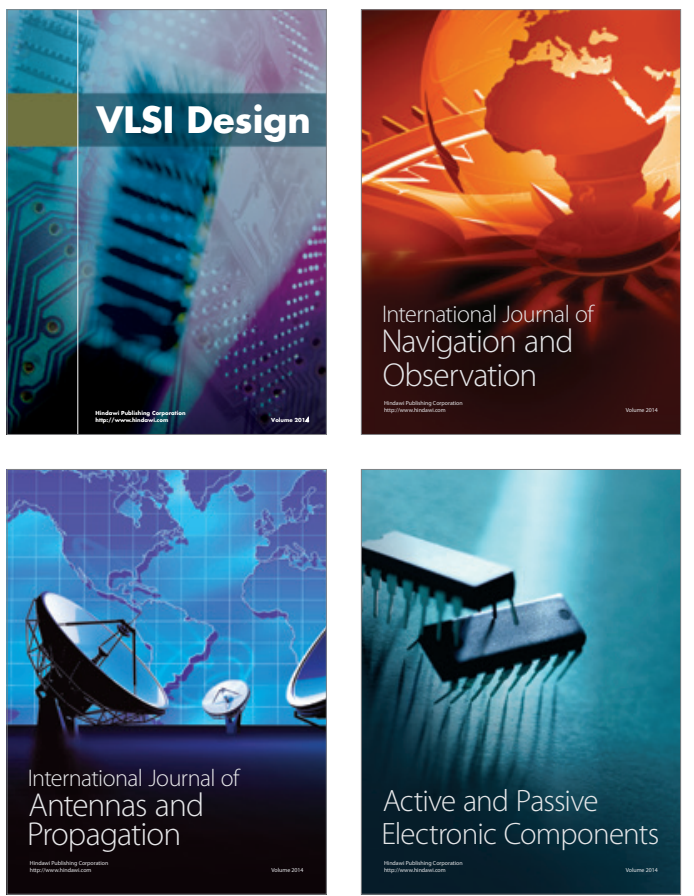
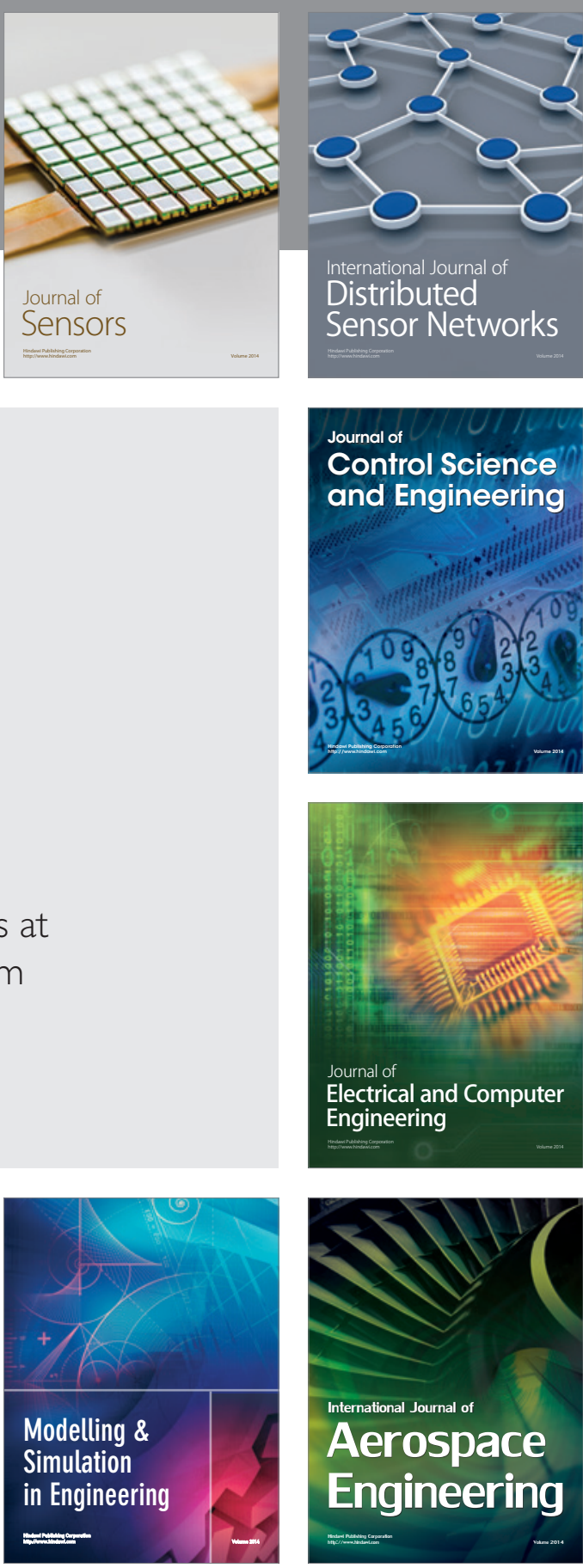

International Journal of

Distributed

Sensor Networks

Journal of

Control Science

and Engineering
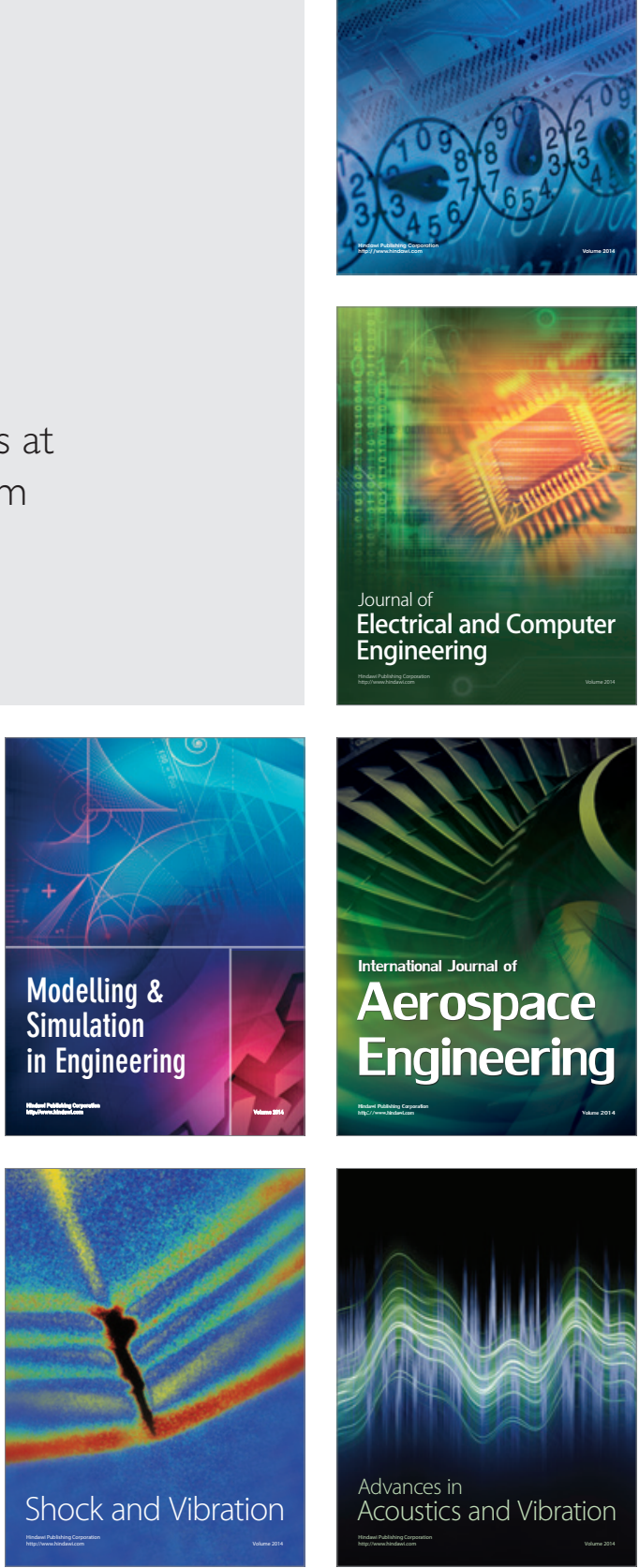